Mauricio Teixeira Leite de Vasconcellos 1,2 Luiz Antonio dos Anjos 2,3

\section{Taxa de adequação (ingestão/requerimento) de energia como indicador do estado nutricional das famílias: uma análise crítica dos métodos aplicados em pesquisas de consumo de alimentos}

\author{
Energy adequacy ratio (intake/requirements) as \\ an indicator of household nutritional assessment: \\ a critical analysis of methods applied to food \\ consumption surveys
}

\footnotetext{
1 Departamento de Metodologia, Diretoria de Pesquisas, Fundação Instituto Brasileiro de Geografia e Estatística. Av. República do Chile 500 10 o andar, Rio de Janeiro, $R J$ 20031-170, Brasil. mtlv@ibge.gov.br

2 Centro de Estudos da Saúde do Trabalhador e Ecologia Humana, Escola Nacional de Saúde Pública, Fundação Oswaldo Cruz. Rua Leopoldo Bulhões 1480 Rio de Janeiro, RJ 21041-210, Brasil. anjos@ensp.fiocruz.br 3 Laboratório de Avaliação Nutricional e Funcional, Departamento de Nutrição Social, Universidade Federal Fluminense. Rua São Paulo 30, 4o andar Niterói, RJ 24040-110, Brasil.
}

\begin{abstract}
The authors discuss problems associated with the use of the energy adequacy ratio as an indicator of nutritional status in population-based studies derived from household food consumption surveys. They describe the use of food consumption data to estimate family energy intake and to compare it among families and with family requirements to determine the family energy adequacy ratio. The article also discusses the use of international recommendations for human energy requirements and their limitations, based on the lack of consideration of nonfood conditions, adaptive mechanisms, inadequacy of estimates of the baseline metabolism rate, and the lack of information to evaluate energy expenditure in non-work physical activities in the household surveys consulted. To illustrate the discussion, the authors use data from the National Family Budget Survey (ENDEF) conducted by the Brazilian Census and Statistics Bureau (IBGE) in 1974-1975. The article concludes that the main use of the adequacy ratio is to identify families suffering food restriction.
\end{abstract}

Key words Nutritional Evaluation; Nutritional Status; Caloric Intake; Food Consumption; Energy Requirements

Resumo O artigo discute os problemas associados ao uso da taxa de adequação de energia como um indicador do estado nutricional, em estudos populacionais derivados de pesquisas domiciliares de consumo de alimentos. Para tanto, descreve o tratamento dos dados de consumo alimentar para estimar a ingestão familiar de energia e compará-la entre famílias, e com os requerimentos da família para determinar a taxa de adequação familiar de energia. $O$ artigo discute, também, uso das recomendações internacionais sobre os requerimentos humanos de energia e suas limitações decorrentes da não-consideração das condições não-alimentares, dos mecanismos de adaptação, da inadequação das estimativas da taxa metabólica basal e da insuficiência de informações para avaliar o gasto energético das atividades físicas não-laborais nas pesquisas domiciliares consideradas. Para exemplificar aspectos da discussão desenvolvida, os dados do Estudo Nacional da Despesa Familiar (ENDEF) realizado pelo IBGE, em 1974-1975, são usados. O artigo conclui que o uso principal da taxa de adequação é na identificação das famílias que sofrem de restrição alimentar.

Palavras-chave Avaliação Nutricional; Estado Nutricional; Ingestão Calórica; Consumo de Alimentos; Necessidade Energética 


\section{Introdução}

Os esforços para elaboração de uma metodologia de pesquisa para a determinação das condições nutricionais da população humana datam do início deste século. Em 1932, na conferência realizada pela Organização de Saúde da Liga das Nações foi descrita uma metodologia para determinação das condições nutricionais agregadas em nível de país (Gibson, 1990), mas somente em 1947, com a Primeira Pesquisa Mundial de Alimentos, realizada pela Organização das Nações Unidas para Alimentação e Agricultura (FAO) durante seu primeiro ano de existência (FAO, 1947), foi iniciada a avaliação de condições nutricionais humanas em escala mundial. Como toda metodologia baseada em informação agregada de países, a pesquisa mundial de alimentos não podia incluir em seus objetivos o estudo da situação nutricional desagregada em regiões e estratos dos países, para apoiar o planejamento alimentar das nações. Cientes disso, a FAO e a Organização Mundial da Saúde (criada em 1948) organizaram "os primeiros comitês para a elaboração de normas sobre a execução e análise de inquéritos nutricionais" (Viacava et al., 1983:9).

A primeira pesquisa domiciliar brasileira, de abrangência nacional, que objetivava mensurar as condições nutricionais da população foi o Estudo Nacional da Despesa Familiar (ENDEF), realizado na década de 70 pela Fundação Instituto Brasileiro de Geografia e Estatística (IBGE), contando com o apoio da FAO (Vasconcellos, 1983a). O ENDEF foi uma pesquisa de consumo alimentar e orçamentos familiares que coletou informações de cerca de 55.000 domicílios, entre agosto de 1974 e agosto de 1975, e que foi concebida com objetivos múltiplos, dentre os quais destaca-se a análise da situação nutricional, tendo por base um quadro de orçamentos familiares, e que teve como principal estimador do estado nutricional das famílias a taxa de adequação, ou seja, a razão entre a ingestão familiar e os requerimentos nutricionais.

Se, por um lado, a metodologia de pesquisa usada no ENDEF incorporou técnicas para corrigir as diferenças observadas entre as famílias, como sua dimensão e composição por sexo e idade, e dentro das famílias, como as diferenças de ritmo alimentar e de participação das pessoas às refeições nos dias laborais e de descanso, por outro lado, sua metodologia limitou a mensuração da ingestão familiar à parcela derivada dos alimentos retirados do estoque familiar, trazendo interferências na avaliação do estado nutricional.
A essas dificuldades do método de coleta das informações de consumo alimentar, somam-se os problemas de uso das recomendações internacionais sobre requerimentos energéticos, alguns derivados do volume e tipo de informação requerida e outros causados pela ausência de diretrizes. No primeiro caso, estão as dificuldades de coletar, ao longo de sete dias, as informações sobre todas as atividades físicas de 343.033 pessoas de alguma forma ligadas às famílias entrevistadas. No segundo caso, não se encontram recomendações internacionais para tratar as condições não-alimentares que afetam a saúde e os mecanismos de adaptação que alteram o valor do requerimento energético. Essas limitações, que interferem tanto nas estimativas da ingestão quanto nas dos requerimentos, combinam-se na estimação das taxas de adequação de energia das famílias e influem na avaliação nutricional da população. Nesse sentido, é importante identificar e discutir essas limitações para poder usar esse indicador do estado nutricional de forma mais adequada. O presente artigo objetiva, portanto, explicitar e discutir os problemas de cálculo da taxa de adequação de energia no que tange à metodologia de tratamento dos dados de consumo alimentar, bem como no que se refere ao uso das recomendações internacionais sobre os requerimentos de energia, tendo, sempre que possível, como base de dados empíricos os coletados no ENDEF.

\section{Ingestão alimentar de energia}

Diversos métodos são propostos na literatura para quantificar a ingestão alimentar de energia de uma pessoa ou de um domicílio (Gibson, 1990). A metodologia de pesquisas de consumo alimentar, que foi utilizada no ENDEF, combina o método do registro alimentar com os de coleta de orçamentos familiares por entrevista ao longo de sete dias consecutivos.

Nessa metodologia de pesquisa, o entrevistador, em princípio, faz tantas visitas quantas sejam as refeições principais da família para: pesar os alimentos que serão consumidos na refeição seguinte; pesar os alimentos comprados no dia, exceto os industrializados que já têm peso líquido na embalagem; e obter o peso dos resíduos, sobras ou desperdícios.

Além das informações alimentares, geralmente obtêm-se, também, dados demográficos (sexo, idade, relação com o chefe da família, escolaridade, migração); antropométricos (fundamentalmente, massa corporal e estatura); de presença às refeições; de condições fisiológicas 
especiais (gestação e lactação); e de despesas, emprego e renda. Esse conjunto de dados primários permite, entre outros usos, calcular os requerimentos de energia para manutenção do metabolismo basal, crescimento das crianças, gestação e atividades físicas necessárias ao exercício das atividades econômicas. Não tem, no entanto, elementos que permitam estimar os gastos energéticos das atividades físicas de lazer (são raras as pesquisas que incluem o levantamento do orçamento de tempo) e os acréscimos relativos às condições não-alimentares (para os quais não existem parâmetros internacionais).

Para estimar a ingestão alimentar, diversos passos são necessários: (1) calcular o peso da parte comestível dos alimentos consumidos e desperdiçados, que corresponde ao peso do alimento tal como é comprado menos o peso dos resíduos não-comestíveis (cascas, ossos, caroços, etc.); (2) estimar, a partir da mediana das razões entre peso total e peso do resíduo, nos registros onde ambos existam, os coeficientes de resíduo dos alimentos, e usá-los para calcular o peso da parte comestível de alimentos nos casos em que os pesos de resíduo não foram observados (esses coeficientes refletem as práticas culinárias da população pesquisada, sendo distintos dos existentes nas tabelas de composição dos alimentos, que são derivados de pequenas amostras e obtidos em laboratório); (3) transformar os pesos das partes comestíveis (itens 1 e 2) em energia e nutrientes, por meio de tabelas de composição química de alimentos; (4) converter as preparações culinárias, em peso da parte comestível dos ingredientes básicos, por meio de receitas médias, derivadas das receitas utilizadas pelas famílias e levantadas ao longo do trabalho de coleta; (5) transformar os pesos de partes comestíveis dos ingredientes básicos em energia e nutrientes, usando tabelas de composição; (6) agregar os dados de energia e nutrientes consumidos e desperdiçados (itens 3 e 5) no nível familiar, para estimar a ingestão de energia e nutrientes pela diferença entre consumo e desperdício.

A ingestão, assim estimada, reflete os valores nutricionais dos alimentos ingeridos pelos membros da família, empregados, hóspedes e convidados, durante a semana de pesquisa, e não é diretamente comparável entre famílias, nem com os requerimentos energéticos semanais da família. Para torná-la comparável entre as famílias (ou grupos de famílias) é necessário corrigir as diferenças de ritmo alimentar das pessoas e as diferenças de tamanho e composição (sexo e idade) das famílias.
A estratégia de correção das diferenças de ritmo alimentar foi proposta por François (1970) e consiste em determinar, para a população em estudo, a ponderação ou importância energética (energia consumida na refeição em relação ao total consumido no dia) de cada refeição em cada ritmo alimentar, para atribuir a cada pessoa, as ponderações das refeições em que de fato participou. A unidade dessa escala é atribuída àqueles que, em um dia, participam em casa de todas as refeições de seu ritmo alimentar. Na ausência de hóspedes e convidados e para uma família onde todos os seus membros fizeram todas as refeições em casa durante a semana de pesquisa, o valor total dessa escala é igual ao tamanho da família multiplicado pelo número de dias de pesquisa.

No ENDEF, a aplicação dessa estratégia de normalização conduziu às ponderações das refeições em cada um dos quinze ritmos alimentares encontrados (Tabela 1). Pode-se observar que apenas dois ritmos alimentares, café-almoço-jantar, mais freqüente entre os adultos, e café-almoço-lanche-jantar, mais comum entre crianças, representam mais de 95,5\% (89.228.260 em 93.413.178 habitantes cujo ritmo alimentar pode ser observado) do total de ocorrências de cada ritmo alimentar, o que significa que essa escala já produziria bons resultados se apenas as ponderações das refeições desses dois ritmos fossem precisas (Vasconcellos, 1983b).

Para cada pessoa em cada dia de pesquisa, é determinado o ritmo alimentar e o índice de presença às refeições, que é igual à soma das ponderações das refeições que fez em casa. A soma, ao longo da semana de pesquisa, dos índices diários de uma pessoa multiplicado por seu requerimento diário de energia fornece o requerimento semanal corrigido da pessoa, que corresponde à parcela dos requerimentos semanais que deve ser satisfeita pela ingestão de alimentos em casa. A soma dos requerimentos semanais corrigidos (pela presença às refeições) de todas as pessoas da família fornece o requerimento familiar corrigido, que é diretamente comparável a ingestão familiar na semana de pesquisa.

A soma, para todas as pessoas da família, dos índices semanais de presença às refeições fornece o total de consumidores dos alimentos ingeridos na semana. Assim, a divisão da ingestão semanal por este total de consumidores gera uma medida da ingestão por pessoa e por dia, que, no ENDEF, foi chamada de ingestão por comensal-dia. Para tornar a ingestão por comensal-dia comparável entre as famílias, é necessário normalizar as diferenças de sexo e idade da pessoa média (o comensal-dia) de cada 
Ponderação das refeições e freqüência média na população brasileira, segundo os ritmos alimentares.

\begin{tabular}{|c|c|c|c|c|c|}
\hline \multirow[t]{2}{*}{ Ritmos alimentares } & \multicolumn{4}{|c|}{ Ponderação das refeições } & \multirow[t]{2}{*}{ Freqüência média* } \\
\hline & Café & Almoço & Lanche & Jantar & \\
\hline \multicolumn{6}{|l|}{ Uma refeição por dia } \\
\hline Café & 1,00 & - & - & - & 117.634 \\
\hline Almoço & - & 1,00 & - & - & 113.061 \\
\hline Lanche & - & - & 1,00 & - & 4.125 \\
\hline Jantar & - & - & - & 1,00 & 114.909 \\
\hline \multicolumn{6}{|l|}{ Duas refeições por dia } \\
\hline Café e almoço & 0,32 & 0,68 & - & - & 933.705 \\
\hline Café e lanche & 0,46 & - & 0,54 & - & 13.683 \\
\hline Café e jantar & 0,46 & - & - & 0,54 & 409.687 \\
\hline Almoço e lanche & - & 0,55 & 0,45 & - & 14.061 \\
\hline Almoço e jantar & - & 0,55 & - & 0,45 & 1.300 .688 \\
\hline Lanche e jantar & - & - & 0,46 & 0,54 & 18.483 \\
\hline \multicolumn{6}{|l|}{ Três refeições por dia } \\
\hline Café, almoço e lanche & 0,22 & 0,57 & 0,21 & - & 745.792 \\
\hline Café, almoço e jantar & 0,18 & 0,49 & - & 0,33 & 48.613 .914 \\
\hline Café, lanche e jantar & 0,24 & - & 0,11 & 0,65 & 134.579 \\
\hline Almoço, lanche e jantar & - & 0,49 & 0,18 & 0,33 & 264.511 \\
\hline \multicolumn{6}{|l|}{ Quatro refeições por dia } \\
\hline Café, almoço, lanche e jantar & 0,16 & 0,43 & 0,11 & 0,30 & 40.614 .346 \\
\hline
\end{tabular}

Adaptado de Vasconcellos (1983b)

* A freqüência média corresponde à estimativa do número, médio na semana, de habitantes, da população residente brasileira em 01/02/75 (96.517.922), com cada ritmo alimentar, considerando que os moradores total ou parcialmente ausentes durante a semana de pesquisa, para os quais não foi determinado o ritmo alimentar, correspondem a 3.104.744 habitantes (3,2\% da população).

família. Para isso, é sugerido construir uma escala de equivalente adulto, baseada nos requerimentos de energia por sexo e idade, atribuindo a unidade da escala ao adulto de referência.

Segundo Vasconcellos (1983b), uma das primeiras escalas de equivalente adulto foi proposta por Engel, em 1883, tomando por base a evolução da massa corporal ao longo do crescimento (Tabela 2). Ainda segundo Vasconcellos (1983b), outras escalas de adulto equivalente foram elaboradas: (a) Atwater, no início do século XX, estabeleceu uma escala baseada em coeficientes fisiológicos (requerimentos energéticos) e não na massa corporal; (b) com os mesmos princípios de Atwater, foi proposta, em 1934, a escala de Bigwood \& Roost, da Liga das Nações (não apresentada aqui); (c) outra escala (também não apresentada) construída com base na despesa por sexo e idade, foi proposta pelo Instituto de Estatística de Oxford, em 1944; (d) Prais \& Houthakker, em 1955, calcularam outra escala em função da despesa alimentar, obtida em pesquisas de orçamentos familiares no Reino Unido. Na escala elaborada para o ENDEF, foi considerado, como unidade, o homem adulto de 20 a 29 anos, com $63,9 \mathrm{~kg}$ de massa corporal e $1,69 \mathrm{~m}$ de estatura, exercendo uma atividade física moderada e requerendo $2.939 \mathrm{kcal}$ por dia. A partir da mediana da massa corporal por idade e sexo, foram calculados os requerimentos energéticos diários de cada grupo, que, relacionados ao requerimento do adulto de referência, determinaram os valores apresentados na Tabela 2 (Vasconcellos, 1983b). A escala da pesquisa ENDEF foi baseada em requerimentos de energia calculados de acordo com as recomendações internacionais da década de 70 (FAO/WHO, 1973).

A soma, para todas as pessoas da família, do produto do índice semanal de presença às refeições pelo valor da escala de adulto equivalente corresponde ao total de comensais adulto-dia, que é o divisor da ingestão para estimar a ingestão por adulto-dia. A ingestão por adulto-dia é diretamente comparável entre famílias e grupos familiares. 
Escalas de adulto equivalente.

\begin{tabular}{|c|c|c|c|c|c|c|c|c|}
\hline Grupo de idade (anos) & \multicolumn{2}{|c|}{ Engel (1883) } & \multicolumn{2}{|c|}{ Atwater (1905) } & \multicolumn{2}{|c|}{ Prais \& Houthakker (1955) } & \multicolumn{2}{|c|}{ ENDEF (1977) } \\
\hline até 1 & \multicolumn{2}{|c|}{0,30} & \multicolumn{2}{|c|}{0,30} & \multicolumn{2}{|c|}{0,35} & \multicolumn{2}{|c|}{0,30} \\
\hline 1 & \multicolumn{2}{|c|}{0,40} & \multicolumn{2}{|c|}{0,30} & \multicolumn{2}{|c|}{0,52} & \multicolumn{2}{|c|}{0,39} \\
\hline 2 & \multicolumn{2}{|c|}{0,40} & \multicolumn{2}{|c|}{0,39} & \multicolumn{2}{|c|}{0,52} & \multicolumn{2}{|c|}{0,43} \\
\hline 3 & \multicolumn{2}{|c|}{0,40} & \multicolumn{2}{|c|}{0,39} & \multicolumn{2}{|c|}{0,52} & \multicolumn{2}{|c|}{0,48} \\
\hline 4 & \multicolumn{2}{|c|}{0,40} & \multicolumn{2}{|c|}{0,39} & \multicolumn{2}{|c|}{0,52} & \multicolumn{2}{|c|}{0,52} \\
\hline 5 & \multicolumn{2}{|c|}{0,50} & \multicolumn{2}{|c|}{0,50} & \multicolumn{2}{|c|}{0,57} & \multicolumn{2}{|c|}{0,54} \\
\hline 6 & \multicolumn{2}{|c|}{0,50} & \multicolumn{2}{|c|}{0,50} & & & & \\
\hline 7 & & & & & & & & \\
\hline 8 & & & & & & & & \\
\hline 9 & & & & & & & & \\
\hline 10 & & & & & & & & \\
\hline 11 & & & & & & & & \\
\hline 12 & & & & & & & & \\
\hline 13 & & & & & & & & \\
\hline & & & & & $\mathrm{H}$ & M & $\mathrm{H}$ & M \\
\hline 14 & & & & & 0,81 & 0,65 & 0,85 & 0,80 \\
\hline 15 & & & & & 0,81 & 0,65 & 0,90 & 0,80 \\
\hline 16 & & & & & 0,81 & 0,65 & 0,95 & 0,80 \\
\hline 17 & & & & & 0,81 & 0,65 & 0,97 & 0,80 \\
\hline 18 & & & & & 1,00 & 0,88 & 0,99 & 0,80 \\
\hline 19 & & & & & 1,00 & 0,88 & 0,99 & 0,80 \\
\hline & $\mathrm{H}$ & $M$ & $\mathrm{H}$ & $M$ & $\mathrm{H}$ & $M$ & $\mathrm{H}$ & $M$ \\
\hline $20-29$ & 1,00 & 0,85 & 1,00 & 0,80 & 1,00 & 0,88 & 1,00 & 0,80 \\
\hline $30-39$ & 1,00 & 0,85 & 1,00 & 0,80 & 1,00 & 0,88 & 1,00 & 0,80 \\
\hline $40-49$ & 1,00 & 0,85 & 1,00 & 0,80 & 1,00 & 0,88 & 1,00 & 0,80 \\
\hline $50-59$ & 1,00 & 0,85 & 1,00 & 0,80 & 1,00 & 0,88 & 1,00 & 0,80 \\
\hline $60-69$ & 1,00 & 0,85 & 1,00 & 0,80 & 1,00 & 0,88 & 0,90 & 0,70 \\
\hline 70-79 & 1,00 & 0,85 & 1,00 & 0,80 & 1,00 & 0,88 & 0,90 & 0,70 \\
\hline $80+$ & 1,00 & 0,85 & 1,00 & 0,80 & 1,00 & 0,88 & 0,90 & 0,70 \\
\hline
\end{tabular}

Adaptado de Vasconcellos (1983b)

Em resumo, essa metodologia permite que os requerimentos energéticos sejam corrigidos e possam ser comparados às ingestões observadas na pesquisa e que estas sejam normalizadas de diferentes formas até produzir uma medida que possa ser comparada entre famílias.

Para ilustrar as diferentes formas de normalizar a ingestão de energia, os dados do ENDEF são usados para estimar alguns parâmetros das distribuições de ingestão na população brasileira (Tabela 3). A variabilidade total da distribuição de ingestão familiar de energia deriva da variabilidade intrafamiliar (número de dias de pesquisa em cada unidade, número de consumidores, quantidade ingerida por consumi- dor) e entre famílias (variações de quantidade e tipo dos alimentos consumidos), resultando em um coeficiente de variação (CV) de 57,6\%. Ao dividir a ingestão de cada família pelo número de consumidores multiplicado pelo número de dias de pesquisa, obtém-se a ingestão per capita-dia da família, cuja distribuição tem uma variabilidade bem menor $(\mathrm{CV}=45,3 \%)$. Ao considerar, no denominador da ingestão, o número de refeições de cada consumidor e a quantidade média de energia fornecida em cada refeição (medidos pelo índice de presença às refeições), chega-se à ingestão por comensal-dia e reduzem-se, ainda mais, os efeitos intrafamiliares na variabilidade total (que passa a ter $\mathrm{CV}=$ 
Tabela 3

Estimativas dos parâmetros das distribuições, segundo o tipo de ingestão de energia (kcal).

\begin{tabular}{lrrrrr}
\hline Tipo de ingestão de energia & Mínimo & Média & Máximo & DP & CV (\%) \\
\hline Familiar & 1.692 & $72.511,3$ & 668.279 & $41.736,1$ & 57,6 \\
Per capita-dia da família & 92 & $2.163,1$ & 36.393 & 979,5 & 45,3 \\
Por comensal-dia da família & 462 & $2.196,4$ & 6.449 & 647,0 & 29,5 \\
Por adulto-dia da família & 658 & $2.843,5$ & 7.378 & 754,8 & 26,5 \\
\hline
\end{tabular}

Fonte: ENDEF.

DP = desvio-padrão; $C V$ = coeficiente de variação.

29,5\%). Ao acrescentar no denominador uma proxy da quantidade ingerida por comensal, expressa pelo seu escore na escala de adulto equivalente, chega-se ao limite de redução da variabilidade intrafamiliar permitida pelos dados obtidos de acordo com a metodologia descrita: a ingestão por adulto-dia da família, cuja distribuição tem $C V=26,5 \%$. Para anular a variabilidade intrafamiliar há que se medir a distribuição intrafamiliar de alimentos, alterando a unidade de pesquisa da família para o indivíduo.

Apesar das normalizações descritas, a ingestão familiar obtida por essa metodologia ainda padece das limitações comuns às tabelas de composição química dos alimentos. Por extensão ao que indica Gibson (1990) para a ingestão individual, a estimativa da ingestão familiar deve ser encarada como indicador da disponibilidade máxima de nutrientes para os membros do grupo e não como a quantidade efetivamente absorvida e utilizada por eles, visto que não considera a bio-disponibilidade dos alimentos.

\section{Requerimentos de energia}

Há dois métodos básicos para estimar os requerimentos humanos de energia: um é baseado na ingestão média de energia de pessoas supostamente sadias e o outro é derivado do gasto energético do organismo. Até meados dos anos 80 , as recomendações internacionais assumiram a hipótese de que a ingestão de energia de pessoas saudáveis e que viviam uma vida normal correspondiam ao seu requerimento energético. Assim, eram fixados os coeficientes de energia por quilograma de massa corporal, por sexo e grupo etário (FAO/WHO, 1973).

A partir de 85 , os especialistas consideraram que determinar requerimentos a partir de ingestões observadas em pessoas saudáveis era um argumento circular, uma vez que elas não eram, forçosamente, as que mantinham a massa corporal desejável e os níveis ótimos de atividade física (devido a possíveis adaptações fisiológicas e sociais) e, portanto, a saúde em seu sentido amplo (FAO/WHO/UNU, 1985). Dessa forma, esses especialistas enfatizaram nas recomendações de 1985, três aspectos: (1) a manutenção da saúde a longo prazo, acrescentando aos requerimentos energéticos uma provisão para a prática de atividades físicas que visam manter a aptidão física do ponto de vista cardiovascular; (2) a necessidade de incluir uma provisão para a atividade física socialmente desejável, adicionalmente à provisão para a atividade economicamente necessária, que já constava das recomendações de 1973; (3) que o método de cálculo dos requerimentos energéticos deveria, tanto quanto possível, ser baseado nos componentes de gasto energético, a saber: (a) o metabolismo basal, ou seja, a manutenção do funcionamento do organismo em estado de repouso completo; (b) digestão e metabolização dos alimentos; (c) atividades físicas (sejam as de trabalho ou de lazer); e (d) crescimento das crianças, sintetização e manutenção dos tecidos novos e secreção de leite.

Assim, as recomendações internacionais atuais para o cálculo dos requerimentos de energia partem, no caso de pessoas com dez anos ou mais, do metabolismo basal (por ser o maior dos componentes na população em geral) e incluem acréscimos para os demais gastos de energia do ser humano. Para as crianças até nove anos completos de idade, essas recomendações fixam aportes diários recomendados de energia por quilograma de massa corporal, que variam por sexo, idade e grau de desenvolvimento do país, considerando, além do gasto diário para o metabolismo basal, o gasto energético diário para o crescimento e para as atividades físicas.

Para obter o requerimento diário recomendado de energia das crianças até nove anos 
completos de idade, James \& Schofield (1990) fornecem os coeficientes (Tabela 4), expressos em kcal por kg, que devem ser multiplicados pelo valor da massa corporal de referência (não o da observada), por sexo e idade. A comparação dos aportes com e sem atividade física desejável, na Tabela 4, indica que o acréscimo recomendado para considerar o nível de atividade física desejável é de cerca de $5 \%$. A comparação entre os aportes diários dos países menos desenvolvidos com os dos países desenvolvidos fornece o acréscimo para crescimento compensatório nos dois primeiros anos de vida, que corresponde a energia suplementar que as crianças dos países menos desenvolvidos necessitam para restabelecerem-se após infecções intermitentes.

Para as pessoas de dez anos completos ou mais, o cálculo do gasto energético diário é baseado na taxa metabólica basal (TMB), geralmente estimada por meio de equações sugeridas pela FAO, em função do sexo, grupo etário e do valor da massa corporal (Tabela 4). Adicionalmente à TMB, é necessário definir o gasto energético para gestação, lactação e para as atividades físicas desejáveis. O relatório FAO/ WHO/UNU (1985) sugere um aporte adicional de $200 \mathrm{kcal} /$ dia durante a gestação sedentária ou de $285 \mathrm{kcal} /$ dia, se não houver redução da atividade física da mulher durante sua gravidez. Para o período de lactação, as recomendações homologam um procedimento que a FAO já vinha adotando nas pesquisas mundiais de alimentos, ou seja, tratar a mãe e o bebê como uma só unidade e considerar os requerimentos do bebê como correspondentes ao acréscimo energético para secreção do leite.

Para lidar com o gasto energético das atividades físicas desejáveis, duas aproximações são sugeridas (FAO/WHO/UNU, 1985; James \& Schofield, 1990): (1) levantamento das atividades em períodos do dia e uso do índice energético integrado (IEI), calculado como a razão entre o dispêndio energético de uma atividade (tarefa), em um certo período de tempo, e o dispêndio do metabolismo basal, no mesmo período; (2) valores únicos para o dia e uso do nível de atividade física (NAF), calculado como a razão entre a energia total gasta no dia e a TMB para 24 horas, ou seja, uma extensão do IEI quando são consideradas todas as atividades em um período de 24 horas. Caso se tenha um acompanhamento detalhado da atividade pode-se calcular o custo energético de uma atividade minuto a minuto, que quando expresso em função do metabolismo basal minuto recebe o nome de razão de atividade física (RAF). Entretanto, o uso do RAF requer uma riqueza de informação que, normalmente, não se dispõe em pesquisas não-laboratoriais, mas seus valores servem de base para o cálculo do IEI e do NAF.

De um modo geral, o NAF é o caminho mais usado na especificação do dispêndio energético total diário das atividades físicas. James \& Schofield (1990) sintetizaram os valores de NAF, propostos nas recomendações de 1985, para a atividade física desejável de adolescentes e adultos, considerando médias populacionais, e de adultos, levando-se em conta o nível de atividade física da ocupação econômica exercida (Tabela 4).

Ainda segundo James \& Schofield (1990), os requerimentos de sobrevivência correspondem a 1,20 vezes a TMB e são definidos para viver permanentemente na cama. Os de inatividade, 1,27 TMB permitem sair da cama, vestirse e comer, mas pressupõem que outras pessoas comprem e preparem os alimentos, como ocorre com pessoas sem autonomia plena. Os requerimentos de manutenção, 1,40 TMB, permitem vestir-se, lavar-se e realizar três horas de atividade em pé, mas não incluem as atividades socialmente desejáveis ou as ocupacionais. Como as pesquisas de consumo de alimentos, geralmente, não coletam informação suficientemente detalhada das atividades físicas das pessoas, o relatório de 1985 (FAO/WHO/ UNU, 1985) sugere uma aproximação baseada no gasto energético da ocupação econômica exercida e no sexo. Se a ocupação tiver RAF $\leq$ 2,5, a recomendação é atribuir um NAF leve (1,55 TMB, para homens, e 1,56 TMB, para mulheres); se $2,5<\mathrm{RAF}<4,0$ atribui-se NAF moderado (1,78 TMB, para homens, e 1,64 TMB, para mulheres); e, se RAF $\geq 4,0$ atribui-se NAF pesado $(2,10 \mathrm{TMB}$, para homens e 1,82 TMB, para mulheres).

Assim, as alternativas para tratar as atividades físicas derivam do valor da TMB, a qual estão relacionados múltiplos da TMB para cada sexo e atividade (RAF, IEI e NAF), o que permite concluir que a TMB é a maior parcela do requerimento energético diário, exceto para homens com NAF pesado, cujo multiplicador é maior do que dois, razão pela qual o uso das equações de predição da TMB merece reflexão especial em relação a três pontos: (1) o valor de massa corporal (MC) usado; (2) sua aplicação para casos individuais; (3) a universalização de sua aplicação.

Existem diferentes escolhas para os valores de MC, dentre os quais destacam-se: os observados, os de referência e os saudáveis. Cada escolha representa um conceito distinto de requerimento energético e decorre de um objeti- 
Recomendações Internacionais para os requerimentos de energia (kcal).

\begin{tabular}{|c|c|c|c|c|c|c|c|c|}
\hline \multirow{4}{*}{$\begin{array}{l}\text { Grupos de idade } \\
\text { (em anos completos) }\end{array}$} & \multicolumn{8}{|c|}{$\begin{array}{l}\text { Aporte energético diário }(\mathrm{kcal} / \mathrm{kg}) \text { para menores de } 10 \text { anos, por sexo, } \\
\text { grau de desenvolvimento do país e atividade física desejável } 1\end{array}$} \\
\hline & \multicolumn{4}{|c|}{ Homens } & \multicolumn{4}{|c|}{ Mulheres } \\
\hline & \multicolumn{2}{|c|}{ País menos desenvolvido } & \multicolumn{2}{|c|}{ País desenvolvido } & \multicolumn{2}{|c|}{ País menos desenvolvido } & \multicolumn{2}{|c|}{ País desenvolvido } \\
\hline & $\begin{array}{c}\text { Sem } \\
\text { atividade } \\
\text { desejável }\end{array}$ & $\begin{array}{c}\text { Com } \\
\text { atividade } \\
\text { desejável }\end{array}$ & $\begin{array}{c}\text { Sem } \\
\text { atividade } \\
\text { desejável }\end{array}$ & $\begin{array}{l}\text { Com } \\
\text { atividade } \\
\text { desejável }\end{array}$ & $\begin{array}{l}\text { Sem } \\
\text { atividade } \\
\text { desejável }\end{array}$ & $\begin{array}{l}\text { Com } \\
\text { atividade } \\
\text { desejável }\end{array}$ & $\begin{array}{c}\text { Sem } \\
\text { atividade } \\
\text { desejável }\end{array}$ & $\begin{array}{c}\text { Com } \\
\text { atividade } \\
\text { desejável }\end{array}$ \\
\hline 0 & 104 & 109 & 98 & 103 & 104 & 109 & 98 & 103 \\
\hline 1 & 103 & 108 & 99 & 104 & 108 & 113 & 103 & 108 \\
\hline 2 & 99 & 104 & 99 & 104 & 97 & 102 & 97 & 102 \\
\hline 3 & 94 & 99 & 94 & 99 & 90 & 95 & 90 & 95 \\
\hline 4 & 90 & 95 & 90 & 95 & 87 & 92 & 87 & 92 \\
\hline 5 & 87 & 92 & 87 & 92 & 84 & 88 & 84 & 88 \\
\hline 6 & 84 & 88 & 84 & 88 & 79 & 83 & 79 & 83 \\
\hline 7 & 79 & 83 & 79 & 83 & 72 & 76 & 72 & 76 \\
\hline 8 & 73 & 77 & 73 & 77 & 66 & 69 & 66 & 69 \\
\hline 9 & 68 & 72 & 68 & 72 & 59 & 62 & 59 & 62 \\
\hline
\end{tabular}

Taxa metabólica basal (kcal)2 para maiores de 9 anos

Grupos de idade Homens

Mulheres

$\begin{array}{lrr}10-17 & 17,5 \mathrm{MC}+651 & 12,2 \mathrm{MC}+746 \\ 18-29 & 15,3 \mathrm{MC}+679 & 14,7 \mathrm{MC}+496 \\ 30-59 & 11,6 \mathrm{MC}+879 & 8,7 \mathrm{MC}+829 \\ 60 \text { ou mais } & 13,5 \mathrm{MC}+487 & 10,5 \mathrm{MC}+596\end{array}$

Valores propostos para o nível de atividade física (NAF) para maiores de 9 anos, por sexo, grau de desenvolvimento do país e atividade desejável 3

\section{Grupos de idade e atividade física} ocupacional

$$
\text { Homens }
$$

\begin{tabular}{cccc} 
País menos desenvolvido & \multicolumn{2}{c}{ País desenvolvido } \\
Sem & Com & Sem & Com \\
atividade & atividade & atividade & atividade \\
desejável & desejável & desejável & desejável
\end{tabular}

Mulheres

País menos desenvolvido País desenvolvido

$\begin{array}{cccc}\text { Sem } & \text { Com } & \text { Sem } & \text { Com } \\ \text { atividade } & \text { atividade } & \text { atividade } & \text { atividade } \\ \text { desejável } & \text { desejável } & \text { desejável } & \text { desejável }\end{array}$

\begin{tabular}{|c|c|c|c|c|c|c|c|c|}
\hline 10 & 1,74 & 1,76 & 1,74 & 1,76 & 1,59 & 1,65 & 1,59 & 1,65 \\
\hline 11 & 1,67 & 1,72 & 1,67 & 1,72 & 1,55 & 1,62 & 1,55 & 1,62 \\
\hline 12 & 1,61 & 1,69 & 1,61 & 1,69 & 1,51 & 1,60 & 1,51 & 1,60 \\
\hline 13 & 1,56 & 1,67 & 1,56 & 1,67 & 1,47 & 1,58 & 1,47 & 1,58 \\
\hline 14 & 1,49 & 1,65 & 1,49 & 1,65 & 1,46 & 1,57 & 1,46 & 1,57 \\
\hline 15 & 1,44 & 1,62 & 1,44 & 1,62 & 1,47 & 1,54 & 1,47 & 1,54 \\
\hline 16 & 1,40 & 1,60 & 1,40 & 1,60 & 1,48 & 1,52 & 1,48 & 1,52 \\
\hline 17 & 1,40 & 1,60 & 1,40 & 1,60 & 1,50 & 1,52 & 1,50 & 1,52 \\
\hline $18-59$ & 1,40 & 1,82 & 1,40 & 1,66 & 1,40 & 1,67 & 1,40 & 1,60 \\
\hline 60 ou mais & 1,40 & 1,51 & 1,40 & 1,51 & 1,40 & 1,56 & 1,40 & 1,56 \\
\hline Atividade Leve & 1,41 & 1,55 & 1,41 & 1,55 & 1,42 & 1,56 & 1,42 & 1,56 \\
\hline Atividade Moderada & 1,70 & 1,78 & 1,70 & 1,78 & 1,56 & 1,64 & 1,56 & 1,64 \\
\hline Atividade Pesada & 2,01 & 2,10 & 2,01 & 2,10 & 1,73 & 1,82 & 1,73 & 1,82 \\
\hline
\end{tabular}

$M C=$ massa corporal $(\mathrm{kg})$

1 Adaptado de: James \& Schofield (1990).

$2 \mathrm{FAO/WHO/UNU}(1985)$

3 Adaptado de: James \& Schofield (1990). Os valores de NAF correspondem ao gasto energético

de um dia dividido pela taxa metabólica basal de um dia. 
vo específico. O uso dos valores da MC observada, por exemplo, aplica-se quando o objetivo é determinar a demanda de energia proveniente dos alimentos, elemento importante para a seguridade e o abastecimento alimentar de uma população. O uso dos valores de referência, geralmente determinados por sexo, idade e estatura, gera requerimentos definidos em termos biológicos e aplica-se quando o objetivo é determinar requerimentos que assegurem, a todos, tamanho e composição corporais compatíveis com a boa saúde a longo prazo e um nível de atividade física socialmente desejável e economicamente necessário. Os problemas com os valores de referência são a necessidade de dados antropométricos de uma população de referência, por definição sadia, e o fato deles serem definidos apenas em função do sexo, idade e estatura da pessoa, sem levar em conta a composição corporal. O uso dos valores de MC saudável (AIN, 1994) objetiva estabelecer diretrizes dietéticas para uma população e seu uso implica em determinar requerimentos energéticos que servem principalmente para prevenir a obesidade e as complicações para a saúde dela derivadas, além de assegurar um mínimo de reservas energéticas para o organismo. $\mathrm{O}$ principal problema com o conceito de MC saudável reside no fato de ele ter sido definido apenas para adultos de 21 anos completos ou mais, pois os especialistas envolvidos (AIN, 1994) consideram que, para definir os limites de MC saudável para menores de 21 anos, há necessidade de mais estudos para poder lidar com o crescimento e o desenvolvimento de crianças e adolescentes.

O uso do conceito de MC saudável em um adulto parte do princípio de que qualquer valor observado que corresponda a um índice de massa corporal (IMC), definido pela razão entre MC $(\mathrm{kg})$ e o quadrado da estatura $(\mathrm{m})$ - entre 18 e 25 é saudável e, portanto, deve-se usar o valor observado. No caso do valor encontrado de MC estar associado a um IMC inferior a 18, o valor saudável corresponde àquele que geraria, para a estatura observada, um IMC igual a este limite inferior. Analogamente, para valor observado associado a IMC $>25$, deve-se reduzir o valor da MC até que o IMC fique igual a este limite superior.

A recomendação internacional para os valores de MC de referência é utilizar dados disponíveis da população de referência do país ou de uma subpopulação supostamente sadia e, na ausência dessas informações, sugere-se o uso de dados internacionais (James \& Schofield, 1990). Os requerimentos energéticos de uma população, definidos em termos biológi- cos (valores de referência da MC), correspondem a uma redistribuição de energia entre os membros dessa população, quando comparado ao conceito de demanda por energia proveniente dos alimentos (valores observados de $\mathrm{MC}$ ), na medida em que são reduzidos os requerimentos energéticos dos que têm valor de MC acima do valor de referência e aumentados os requerimentos dos de MC inferior ao de referência. O mesmo ocorre com os requerimentos energéticos calculados com valores saudáveis de MC, porém em menor grau, uma vez que são mantidos os valores observados das pessoas cujo IMC não seja inferior a $18 \mathrm{nem}$ superior a 25 .

O uso das equações de predição da TMB para casos individuais deve ser evitado. James \& Schofield (1990) indicam que o coeficiente de variação entre os requerimentos individuais é da ordem de $10 \%$, o que implica uma variação substancial entre as pessoas, e sugerem que, no caso de uma pessoa, o mais preciso é medir a sua TMB. Como, porém, medir a TMB é impraticável na clínica médica, a solução é conviver com erros elevados. Como, no entanto, os erros cometidos ao estimar a TMB de cada pessoa tendem a compensar-se quando o número de pessoas aumenta (lei dos grandes números), a estimação da TMB pelas equações da FAO é aceita no caso de estudos populacionais.

A universalização da aplicação dessas equações de predição da TMB para a espécie humana vem sendo questionada por vários autores em diferentes regiões do mundo, particularmente nos trópicos (Henry \& Rees, 1991; Piers \& Shetty, 1993; Soares \& Shetty, 1988), inclusive em dois estudos brasileiros recentes. Cruz et al. (1999) apresentaram resultados empíricos sobre cinqüenta estudantes universitárias do Rio de Janeiro, mostrando que as equações da FAO superestimaram significativamente a TMB em $80 \%$ dos casos. Wahrlich (2000) mediu a TMB de sessenta mulheres (de 20 a 40 anos, residentes em Porto Alegre) e constatou que as equações de predição superestimam 78,4\% dos casos. A TMB média calculada pelas equações de predição em ambos os estudos, foi $12,2 \%$ maior do que a média medida. Este ponto é de grande importância pois não se limita ao erro cometido na maior parcela do gasto energético: o erro da TMB é multiplicado pelos valores de RAF, IEI ou NAF. Além disso, os valores de RAF, IEI e NAF divulgados internacionalmente foram calculados com base na TMB superestimada e, portanto, podem não ser adequados caso se use o valor da TMB medida, ou seja, talvez seja necessário recalculá-los tomando-se por base o valor medido. 
Numa reunião recente, o International Dietary Energy Consultative Group (IDECG) recomendou que sejam mantidos os princípios gerais de cálculo dos requerimentos de energia e sua definição, contidos no relatório de 1985 (FAO/WHO/UNU, 1985) e que mais dados de TMB sejam coletados para que novas equações de predição sejam calculadas, particularmente para menores de dez anos e maiores de sessenta anos, cujos valores deveriam ser expressos em três classes de idade (60-75; 76-85; e 86 anos ou mais), além da obtenção de mais informações sobre o custo energético de diferentes atividades (para cálculo do NAF) em todos os grupos etários (Butte et al., 1996; Buyckx et al., 1996; Dupont et al., 1996). Além disso, as recomendações internacionais para bebês (até 12 meses incompletos de idade) saudáveis foram consideradas muito altas, sendo sugerida a obtenção de mais informações de gasto energético de bebês de populações com risco de altas taxas de infecção e expostos a outras condições ambientais de risco para determinar a influência desses fatores sobre os requerimentos energéticos (Butte, 1996).

O IDECG identificou, particularmente com relação a crianças e adolescentes (1 a 17 anos completos), que: (1) as recomendações superestimam os requerimentos para crianças abaixo de oito anos completos; (2) as recomendações subestimam, principalmente em áreas rurais de países em desenvolvimento, os requerimentos a partir dos 12 anos; (3) não há garantias para recomendar, para crianças abaixo de dez anos, o acréscimo de $5 \%$ dos requerimentos para permitir um nível de atividade física socialmente desejável (Butte et al., 1996). Reconhece-se, ainda, que há grande diferenciação no estilo de vida e no nível de atividade física de adolescentes e crianças acima de cinco anos, entre os países desenvolvidos e em desenvolvimento, e sugerem que as recomendações para esse grupo sejam baseadas em três níveis de atividade física habitual, similares às do relatório de 1985 (FAO/WHO/UNU, 1985) para adultos.

Com relação aos adultos, apesar do IDECG recomendar a manutenção dos valores de NAF (Buyckx et al., 1996), vários autores têm sugerido uma reavaliação, principalmente após a obtenção de mais informações sobre o gasto energético diário através da técnica da água duplamente marcada (Haggarty et al., 1994; Heini et al., 1996).

Além dos pontos mencionados, existe ainda a influência dos processos de adaptação sobre os requerimentos de energia. Quando o gasto de energia é igual à ingestão de energia, a pessoa encontra-se em equilíbrio energético.
Quando a ingestão de energia é maior (ou menor) que os requerimentos energéticos, iniciase um processo de ajuste dos fatores que determinam o gasto de energia em busca de um novo nível de equilíbrio energético. Esse processo, conhecido como adaptação, pode ser de três tipos (FAO, 1987): (1) social ou comportamental, quando a pessoa aumenta (ou reduz) o seu nível de atividade física, voluntariamente ou não, para compensar o aumento (ou redução) da ingestão de energia, evitando o armazenamento de gordura no corpo (ou o emagrecimento); (2) biológico, quando o aumento (ou diminuição) da massa corporal implica em maior (ou menor) gasto de energia para digestão e metabolização dos alimentos; e (3) metabólico, quando o equilíbrio energético é obtido sem variação na atividade física ou na massa corporal, apenas alterando a forma de metabolizar a energia. Este último tipo de mecanismo de adaptação segue o princípio segundo o qual as necessidades humanas de energia de um determinado nível e padrão de atividade física e massa corporal são fixas (Sukhatme, 1989). Essa adaptação, considerada hereditária, faz com que indivíduos submetidos a estresse nutricional sejam metabolicamente mais eficientes. Existem, entretanto, questionamentos fisiológicos sobre a existência desse tipo de adaptação (Ferro-Luzzi et al., 1997; McNeill et al., 1987; Waterlow et al., 1989).

Os mecanismos de adaptação só funcionam, no entanto, dentro de certos intervalos de variação da ingestão de energia. A um nível muito baixo de ingestão pode não ser possível chegar a um equilíbrio e o organismo definha, eventualmente até a morte. Por outro lado, níveis muito altos de ingestão conduzem à obesidade e a doenças cardiovasculares. Assim, existem dois limiares de sobrevivência entre os quais um equilíbrio energético pode ser alcançado (FAO, 1987). Além disso, dados bem recentes indicam que a composição da dieta (alto ou baixo índice glicêmico) pode influenciar as adaptações fisiológicas, por exemplo na TMB, em homens com sobrepeso (Agus et al., 2000).

Há que se considerar, ainda, que os mecanismos de adaptação têm um custo e que diferentes níveis de equilíbrio energético estão associados a diferentes tipos e graus de custo. A redução do nível de atividade física, por exemplo, pode implicar em redução da capacidade de trabalho ou do lazer necessários para uma qualidade de vida aceitável. A variação da massa corporal pode afetar a taxa de crescimento físico de crianças, aumentar a morbidade (suscetibilidade a infecções, em caso de pesos muito baixos, ou a doenças cardiovasculares, em 
caso de obesidade), além de reduzir a capacidade laboral.

Outro aspecto, para alguns implícito na definição internacional de requerimentos de energia, relaciona-se às condições de saúde e saneamento em que vivem os grupos populacionais e que, de certa forma, justificam as recomendações diferenciadas para países menos desenvolvidos. De fato, a energia disponível para o gasto energético depende da absorção efetiva dos alimentos ingeridos e a absorção depende, inter alia, das condições ambientais e de saúde. Assim, os requerimentos de energia dependem de fatores ou condições não-alimentares.

Condições sanitárias ruins que aumentam os riscos de infecções, por exemplo, podem ser compensadas, até certo ponto, por uma ingestão alimentar maior e mais rica em alguns nutrientes. Condições patológicas, como infecções, também têm relação com a ingestão alimentar, seja pela perda de apetite que reduz a ingestão, seja até pela impossibilidade de ingerir alimentos sólidos. Na prática, no entanto, essas condições não-alimentares são de difícil tratamento e são desconsideradas nas recomendações internacionais. Este fato tem implicações sobre a interpretação das taxas de adequação nutricional, como é discutido mais adiante.

\section{Taxa de adequação}

Nas pesquisas domiciliares sobre consumo de alimentos, a taxa de adequação de energia de uma família é definida como a razão entre a ingestão de energia (observada nas refeições feitas ou preparadas em casa) e o requerimento energético familiar corrigido (pelo índice de presença às refeições). Apesar de todas as correções propostas para a ingestão e para os requerimentos, a taxa de adequação de energia ainda tem limitações de cálculo e de utilização: (1) a falta de dados sobre as atividades físicas não-laborais não permite que seja corretamente determinado o gasto energético dessas atividades, sendo necessário assumir hipóteses ou padrões comportamentais da população (como os que deram origem aos valores de NAF propostos nas recomendações internacionais); (2) a taxa de adequação deriva de uma média na família e, portanto, não permite estimar corretamente o número de pessoas subnutridas, pois a distribuição intrafamiliar de alimentos não é conhecida, podendo haver subnutridos em famílias com bom nível de adequação ou não-subnutridos em famílias com baixo nível de adequação; (3) a ingestão refere-se apenas ao consumo dos alimentos retirados do estoque familiar e a correção feita nos requerimentos individuais, por meio do índice de presença, representa uma correção baseada na ponderação energética média das refeições, o que significa que famílias com hábitos distintos da média observada ou com uma alta incidência de refeições consumidas fora de casa podem ter seu estado nutricional mal determinado por esse indicador; (4) a interpretação da taxa de adequação é também afetada pelo fato de ela ser um indicador das manifestações físicas da inadequação da ingestão, que não leva em conta os mecanismos de adaptação e as condições não-alimentares.

Suponha-se, para facilitar o entendimento do item 4 acima, que foram determinados os requerimentos e a ingestão de cada pessoa e calculadas as suas taxas de adequação. Em princípio, o número de pessoas cujas ingestões de energia, em um dado período de tempo, são menores que seus requerimentos de energia no mesmo período (ou seja, com taxa de adequação $<1$ ), tem tradicionalmente sido interpretado como uma medida da incidência de subnutrição. Entretanto, "subnutrição refere-se, por convenção, às manifestações físicas decorrentes da inadequação nutricional que desvia as medidas antropométricas para valores inferiores aos valores de referência" (FAO, 1987:53). Assim, o uso da taxa de adequação (como o de qualquer outro indicador baseado em ingestões observadas) para medir a incidência de subnutrição significa usar um conceito baseado na entrada para medir um conceito definido por conseqüências ou resultados. Há pelo menos duas razões pelas quais esse conceito de entrada não produz medidas precisas da subnutrição física: (1) a taxa de adequação baixa (ou a ingestão alimentar inadequada) não necessariamente conduz à subnutrição física devido aos mecanismos de adaptação; (2) a subnutrição física pode ocorrer mesmo quando a ingestão de energia é superior ao nível requerido (taxa de adequação > 1) devido a condições não-alimentares inadequadas.

Apesar dos mecanismos de adaptação, a ingestão inferior ao requerimento de fato representa um problema nutricional que pode, se mantido por algum tempo, conduzir aos sinais físicos da subnutrição ou a uma redução do nível de atividade física ou a ambos. Mesmo os casos de redução da atividade física, na busca de um equilíbrio energético que permita fugir da subnutrição física, representam custos, no caso sociais, representados por redução da renda ou a piora da qualidade de vida. 
As condições não-alimentares afetam a taxa de adequação no sentido em que podem provocar uma subestimação da subnutrição física, como pode-se observar na Figura 1, onde está representado, teoricamente, um conjunto de pessoas com um mesmo requerimento de energia, representado por $\mathrm{R}_{\mathrm{m}}$ (a recomendação mínima, calculada sem considerar as condições não-alimentares), que têm distintos níveis de ingestão de energia (representados no eixo das abcissas) e que vivem em condições nãoalimentares distintas (representadas no eixo das ordenadas). $\mathrm{O}$ valor $\mathrm{F}_{0}$ corresponderia ao mínimo aceitável das condições não-alimentares, abaixo do qual não há quantidade adicional de alimentos que permita alcançar um equilíbrio energético.

A curva I, cuja forma e posição são arbitrárias, corresponde ao contorno de isonutrição, onde todos os pontos da curva representam o mesmo nível de gasto energético mínimo, ou seja, qualquer ponto abaixo da curva representa a existência de um problema nutricional (em sentido amplo). Assim, cada ponto na curva I corresponde a uma combinação de ingestão e condições não-alimentares, adequada para cobrir o gasto energético mínimo.

A interpretação tradicional da taxa de adequação de energia conduz a considerar como subnutridas todas as pessoas classificadas nas áreas A e B (ingestão $<\mathrm{R}_{\mathrm{m}}$ ) e como não-subnutridas as pessoas que se encontram nas áreas $\mathrm{C}$ e $\mathrm{D}$. De fato, as pessoas que estão na área $\mathrm{A}$ podem atingir um nível adequado de nutrição apenas com o aumento de sua ingestão, enquanto as pertencentes à área B necessitam da

Figura 1

Relação entre ingestão, condições não-alimentares e requerimento.

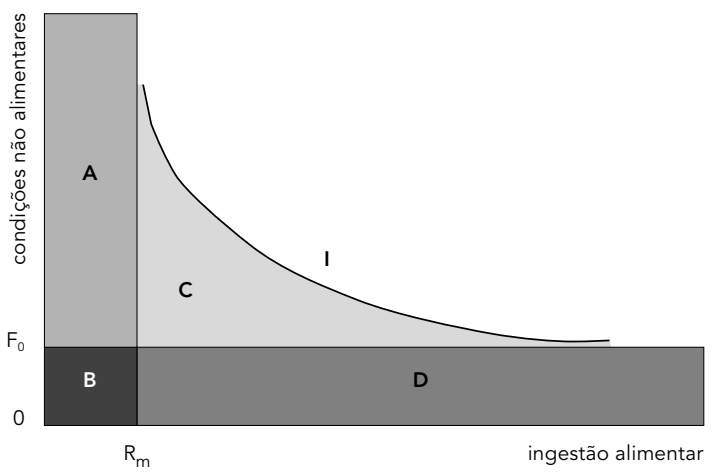

melhora de suas condições não-alimentares, além do aumento de sua ingestão. As localizadas na área $\mathrm{C}$ necessitam, para atingir o equilíbrio entre sua ingestão e seu gasto energético, do aumento em sua ingestão ou da melhora em suas condições não-alimentares. Por fim, para as pessoas classificadas na área $\mathrm{D}$, a única forma de assegurar uma nutrição adequada é através da melhora de suas condições não-alimentares. A proporção de pessoas nas áreas $\mathrm{C}$ e D fornece uma indicação do grau de subestimação da taxa de adequação, quando os requerimentos são calculados sem levar em conta as condições não-alimentares.

O raciocínio feito aplica-se mutatis mutandis à taxa de adequação familiar, o que permite concluir que, apesar dessas limitações, a taxa de adequação de energia (que não leva em conta as condições não-alimentares) de fato estima de forma precisa o contingente de famílias com problemas de restrição alimentar, isto é, com ingestão menor que o requerimento. Esta é, portanto, a interpretação correta dessa medida, que é particularmente importante no contexto da adequação nutricional e do planejamento alimentar.

\section{Considerações Finais}

Ao longo do presente artigo, tentou-se pontuar os problemas observados na estimação da ingestão e dos requerimentos e suas conseqüências para a taxa de adequação de energia das famílias. De um modo geral, todos os problemas identificados pressupõem mais pesquisa: uns demandam a ampliação do conhecimento científico, como os mecanismos de adaptação e o tratamento de condições não-alimentares; outros demandam aprimoramento em suas estimativas, como os valores das tabelas de composição, o gasto energético por atividade física, as equações de predição da TMB e os valores de massa corporal de referência; enquanto um último grupo requer mudanças na metodologia das pesquisas de consumo alimentar, como a determinação do conjunto mínimo de informações para estimar a distribuição intrafamiliar de alimentos e os níveis das atividades físicas não-laborais. O próprio banco de dados do ENDEF poderia ser usado como fonte para quantificar os efeitos de alguns dos pontos indicados, o que poderia facilitar o planejamento de pesquisas futuras. 


\section{Referências}

AGUS, M. S. D.; SWAIN, J. F.; LARSON, C. L.; ECKERT, E. A. \& LUDWIG, D. S., 2000. Dietary composition and physiologic adaptations to energy restriction. American Journal of Clinical Nutrition, 71: 901-907.

AIN (American Institute of Nutrition), 1994. Report of the American Institute of Nutrition Steering Committee on Health Weight. Journal of Nutrition, 124:2240-2243.

BUTTE, N. F., 1996. Energy requirements of infants. European Journal of Clinical Nutrition, 50(Sup. 1):S24-S36.

BUTTE, N. F.; HENRY, C. J. K. \& TORUN, B., 1996. Report of the working group on energy requirements of infants, children and adolescents. European Journal of Clinical Nutrition, 50(Sup. 1):S188S189.

BUYCKX, M.; DUPONT, J. L.; DURNIN, J. L.; FERROLUZZI, A.; ROBERTS, S. B.; SCHÜRCH, B. \& SHETTY, P. S., 1996. Report of the working group on general principles of assessing energy requirements. European Journal of Clinical Nutrition, 50(Sup. 1):S186-S187.

CRUZ, C. M.; SILVA, A. F. \& ANJOS, L. A., 1999. A taxa metabólica basal é superestimada pelas equações preditivas em universitárias do Rio de Janeiro. Archivos Latinoamericanos de Nutrición, 49:232237.

DUPONT, J. L.; DURNIN, J. V. G. A.; FERRO-LUZZI, A.; ROBERTS, S. B.; SCHÜRCH, B. \& SHETTY, P. S., 1996. Report of the working group on energy requirements of older individuals. European Journal of Clinical Nutrition, 50(Sup. 1):S192.

FAO (Food and Agriculture Organization of the United Nations), 1947. The First World Food Survey. Rome: FAO.

FAO (Food and Agriculture Organization of the United Nations), 1987. The Fifth World Food Survey. Rome: FAO.

FAO(Food and Agriculture Organization of the United Nations)/WHO (World Health Organization), 1973. Energy and Protein Requirements: Report of a Joint FAO/WHO ad hoc Expert Consultation. Nutrition Meeting Report Series 52. Rome: FAO.

FAO(Food and Agriculture Organization of the United Nations)/WHO(World Health Organization)/UNU (University of the United Nations), 1985. Energy and Protein Requirements: Report of a Joint FAO/ WHO/UNU ad hoc Expert Consultation. Technical Report Series 724. Geneva: WHO.

FERRO-LUZZI, A.; PETRACCHI, C.; KURIYAN, R. \& KURPAD, A. V., 1997. Basal metabolism of weightstable chronically undernourished men and women: Lack of metabolic adaptation and ethnic differences. American Journal of Clinical Nutrition, 66:1086-1093.

FRANÇOIS, P. J., 1970. Food Consumption Surveys: Study of a general formula for the estimation of per caput, household and group consumption. FAO Nutrition Newsletter, 8:35-58.

GIBSON, R. S., 1990. Principles of Nutritional Assessment. New York: Oxford University Press.

HAGGARTY, P.; McNEILL, G.; MANNEH, M. K.; DAVIDSON, L.; MILNE, E.; DUNCAN, G. \& ASHTOH, J.,
1994. The influence of exercise on the energy requirements of adult males in the UK. British Journal of Nutrition, 72:799-813.

HEINI, A. F.; MINGHELLI, G.; DIAZ, E.; PRENTICE, A. M. \& SCHUTZ, Y., 1996. Free-living energy expenditure assessed by two different methods in rural Gambian men. European Journal of Clinical Nutrition, 50:284-289.

HENRY, C. J. K. \& REES, D. G., 1991. New predictive equations for the estimation of basal metabolic rate in tropical peoples. European Journal of Clinical Nutrition, 45:177-185.

JAMES, W. P. T. \& SCHOFIELD, E. C., 1990. Human Energy Requirements: A Manual for Planners and Nutritionists. New York: Food and Agriculture Organization/Oxford University Press.

McNEILL, G.; RIVERS, J. P. W.; PAYNE, P. R.; DE BRITTO, J. J. \& ABEL, R., 1987. Basal metabolic rate of Indian men: No evidence of metabolic adaptation to a low plane of nutrition. Human Nutrition: Clinical Nutrition, 41C:473-483.

PIERS, S. \& SHETTY, P. S., 1993. Basal metabolic rates of Indian women. European Journal of Clinical Nutrition, 47:586-591.

SOARES, M. J. \& SHETTY, P. S., 1988. Validity of Schofield's predictive equations for basal metabolic rates in Indians. Indian Journal of Medical Research, 88:253-260.

SUKHATME, P. V., 1989. Nutritional adaptation and variability. European Journal of Clinical Nutrition, 43:75-87.

VASCONCELLOS, M. T. L., 1983a. Metodologia do Estudo Nacional da Despesa Familiar: Objetivos, Descrição e Metodologia Usada no ENDEF. ENDEF, v. 3 (Publicações especiais), t. 4, pt. 1. Rio de Janeiro: Fundação Instituto Brasileiro de Geografia e Estatística.

VASCONCELLOS, M. T. L., 1983b. Metodologia do Estudo Nacional da Despesa Familiar: Núcleo do Banco de Informações ENDEF. ENDEF, v. 3 (Publicações especiais), t. 4, pt. 2. Rio de Janeiro: Fundação Instituto Brasileiro de Geografia e Estatística.

VIACAVA, F.; FIGUEIREDO, C. M. P. \& OLIVEIRA, W. A., 1983. A Desnutrição no Brasil: Uma Análise do Estudo Nacional da Despesa Familiar (IBGE 74/75) para o Nordeste, Estado de São Paulo e Estado do Rio de Janeiro. Petrópolis: Financiadora de Estudos e Projetos/Editora Vozes.

WAHRLICH, V., 2000. Taxa Metabólica Basal em Mulheres Residentes em Porto Alegre, Rio Grande do Sul. Dissertação de Mestrado, Rio de Janeiro: Instituto de Nutrição, Universidade Federal do Rio de Janeiro.

WATERLOW, J. C.; JAMES, W. P. T. \& HEALY, M. J. R., 1989. Nutritional adaptation and variability. Three commentaries on the paper by Professor P. V. Sukhatme. European Journal of Clinical Nutrition, 43:203-210. 